\title{
Hetero-Diels-Alder reactions of new sulfonylsulfines generated from $\alpha$-substituted methylsulfones
}

\author{
Hélène Dentel, Jean-François Lohier and Mihaela Gulea*
}

Laboratoire de Chimie Moléculaire et Thioorganique, UMR CNRS 6507, INC3M, FR 3038, ENSICAEN, Université de Caen Basse Normandie, 6, Bd. Maréchal Juin, 14050 Caen, France

E-mail: mihaela.gulea@ensicaen.fr

Dedicated to Professor Heinz Heimgartner on the occasion of his $70^{\text {th }}$ birthday

\begin{abstract}
Hetero-Diels-Alder reaction with sulfines generated in situ from methylsulfones 5a-e substituted in $\alpha$-position with a phosphonyl, carboxyl, carboxyoxazolidinyl, pyridyl, or a quinolyl substituent, respectively, led to new highly functionalized thiopyrans cycloadducts. When in the substrates (i.e. 5a-c) the sulfonyl group and the second substituent on the methylene carbon have comparable electronwithdrawing effect, a mixture of cis and trans isomers of the corresponding cycloadducts is obtained with low to moderate selectivities. In the case of substrates $\mathbf{5 d}$ and 5e, due to the strong electronwithdrawing effect of the sulfonyl compared to the pyridyl or quinolyl groups, a single isomer is obtained for cycloadducts $\mathbf{7 d}$ and $\mathbf{7 e}$. The stereochemical arrangements in the two cycloadducts resulting from $\mathbf{5 b}$ and $\mathbf{5 d}$ (carboxylate and pyridine derivatives) have been determined by single-crystal X-ray analysis and showed that the trans isomer was favored in both cases.
\end{abstract}

Keywords: thia-Diels-Alder reaction, sulfones, sulfines, thiopyran $S$-oxide

\section{Introduction}

Although less explored than the oxa and aza hetero-Diels-Alder (HDA) reactions, the thiaDiels-Alder version represents an efficient and atom-economical synthetic method to obtain dihydrothiopyrans, which were then used as precursors for the synthesis of some bioactive molecules, ${ }^{1}$ in particular thiosugars. ${ }^{2}$ In the main reported cases, the functionalities required in the target molecule (obtained via a thia-HDA step) were introduced directly in the cycloaddition step, as they belong to the thiocarbonyl heterodienophile or to the diene, or they were introduced by subsequent reactions in particular on the cycloadduct double bond. 
In some recent publications, in order to introduce new functionalities in the thiopyran structure, the cycloadduct was oxidized into the corresponding sulfoxide and a nucleophile was introduced via a Pummerer reaction. ${ }^{1 \mathrm{k}, 3}$ For this purpose, in our laboratory, three substrates have been selected, namely sulfoxides 3a-c, which can be obtained by HDA reaction between dithioesters 1a-c and 1,3-butadiene, and subsequent $S$-oxidation of the resulting cycloadducts 2 ac (Scheme 1). This required a selective oxidation of the endocyclic sulfur atom of 2 versus the exocyclic one. The oxidation of $\mathbf{2 a}$ and $\mathbf{2} \mathbf{b}$ by $m$-CPBA at $-78{ }^{\circ} \mathrm{C}$ afforded sulfoxides $\mathbf{3 a}{ }^{3 a}$ and $\mathbf{3 b},{ }^{4}$ respectively, as the major products ( $>80 \%$ in the crude reaction mixture), however together with a little amount of the isomer 3'. In the case of the pyridine derivative $\mathbf{2 c}$, the oxidation by $m$-CPBA led to a complexe mixture. ${ }^{4}$ To avoid these problems, a solution is to access directly compound 3 by using as heterodienophile the sulfine 4 , which could be obtained by $S$-oxidation of dithioester 1 (Scheme 1). ${ }^{5}$ However, it was described that oxidation by $m$-CPBA of dithioester 1c led to the corresponding sulfine, which was unstable and easily rearranged and decomposed into $S$-methyl 2-pyridylthiocarboxylate [2-PyC(O)SMe]. ${ }^{6}$ Indeed, similar results were obtained also with the phosphonate and carboxylate derivatives, and the corresponding thiocarboxylates $\mathrm{ZC}(\mathrm{O}) \mathrm{SMe}$ have been detected in the mixture of the reaction products.

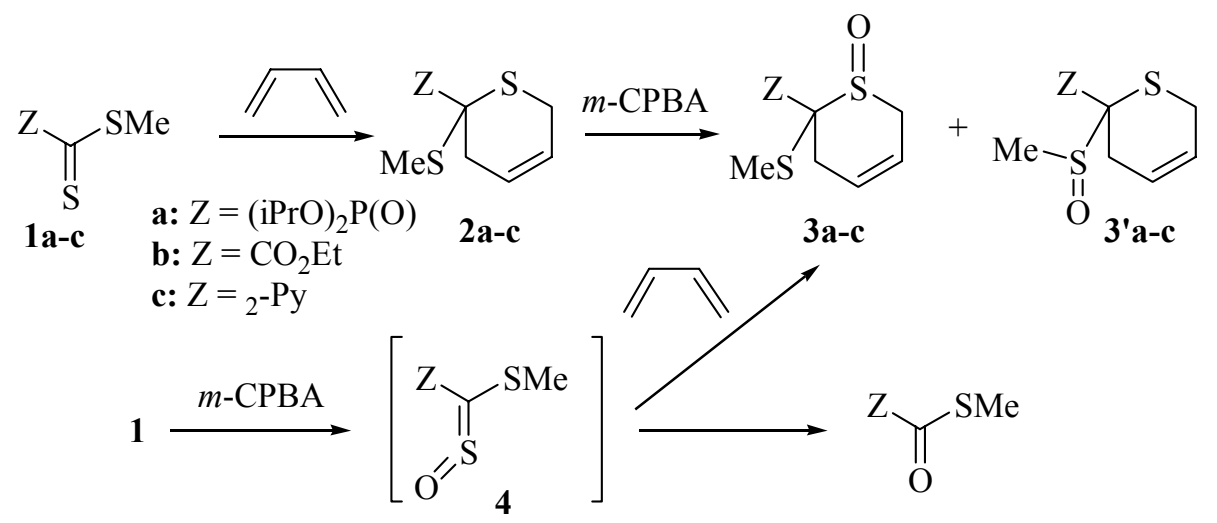

\section{Scheme 1}

Therefore, the use as heterodienophiles of sulfonylsulfines 6 was envisaged (Scheme 2). This type of sulfines bearing two electronwithdrawing groups cannot be isolated because of their high reactivity, but they can be generated as already described in the literature, from the corresponding $\alpha$-substituted methylsulfones $\mathbf{5}$ (as doubly activated methylene compounds) and thionyl chloride $\left(\mathrm{SOCl}_{2}\right)$, in the presence of a base, ${ }^{5,7}$ then trapped in situ by a HDA reaction with a diene. ${ }^{8}$

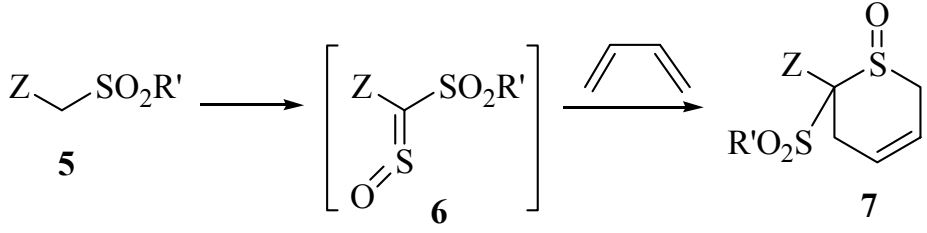

\section{Scheme 2}


This paper describes our results obtained in the thia-Diels-Alder reaction of sulfonylsulfines generated from $\alpha$-substituted methylsulfones. The newly obtained cycloadducts represent highly functionalized thiopyrans and interesting substrates for Pummerer reactions.

\section{Results and Discussion}

For this study we selected five structures of methylsulfones $5\left(\mathrm{ZCH}_{2} \mathrm{SO}_{2} \mathrm{Ph}\right)$ in which the substituent $\mathrm{Z}$ is a phosphonyl, carboxylate, carboxyoxazolidinone, 2-pyridyl, or a 2-quinolyl group (Figure 1). The corresponding dithioesters $\left(\mathrm{ZCS}_{2} \mathrm{Me}\right)$ having the same $\mathrm{Z}$ substituents have already been used in our laboratory in HDA reaction. ${ }^{1 \mathrm{~h}-\mathrm{j}, 4}$ Sulfonyl methylphosphonate $\mathbf{5 a}$ was obtained by oxidation with $m$-CPBA of the corresponding sulfide [(iPrO $)_{2} \mathrm{P}(\mathrm{O}) \mathrm{CH}_{2} \mathrm{SPh}$, which were prepared from diisopropyl $\alpha$-hydroxymethylphosphonate $\left[(\mathrm{iPrO})_{2} \mathrm{P}(\mathrm{O}) \mathrm{CH}_{2} \mathrm{OH}\right]$ by a reported procedure. ${ }^{9}$ Sulfones $\mathbf{5} \mathbf{b}$-e were prepared from the corresponding chlorides $\left(\mathrm{ZCH}_{2} \mathrm{Cl}\right)$ and sodium benzenesulfinate $\left(\mathrm{PhSO}_{2} \mathrm{Na}\right)$ by a reported procedure. ${ }^{10}$
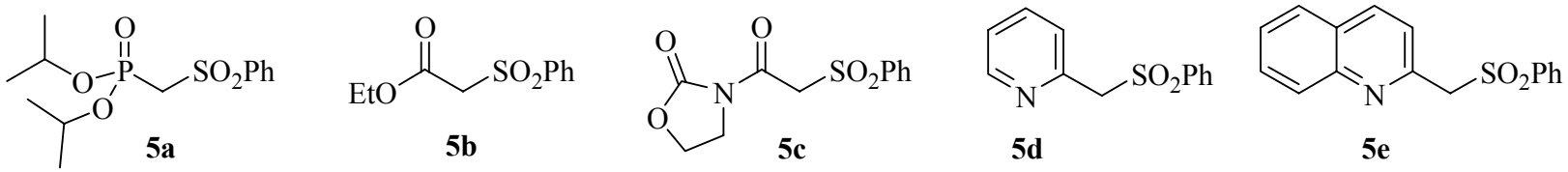

\section{Figure 1}

It is known that when the substituents at the sulfine carbon are different, the sulfine exists as two geometrical isomers and the Diels-Alder reaction is stereospecific, as the geometry of the sulfine $(Z / E)$ is retained in the cycloadduct (cis/trans). In our cases, $Z / E$ (for the sulfine) and cis/trans (for the cycloadduct 7) indicate the relative position between the sulfonyl group and the oxygen of the thiocarbonyl $S$-oxide function (Scheme 3). Sulfones 5 reacted with thionyl chloride in the presence of a base (triethylamine or 2,6-lutidine) to generate the corresponding non-isolable sulfines, which reacted in situ with 2,3-dimethyl-1,3-butadiene or 1,3-butadiene to afford the expected cycloadducts 7 .

The reaction between the sulfine prepared from phenylsulfonyl methylphosphonate 5a, $\mathrm{SOCl}_{2}$ and triethylamine, and 2,3-dimethyl-1,3-butadiene was complete, leading to product 7a as a mixture of trans and cis isomers, in $75 \%$ yield (Table 1, entry 1 ). The ratio of the two isomers (major/minor) was 67/33, but the corresponding relative configuration was not assigned. Similarly, the reaction with 1,3-butadiene afforded cycloadduct $\mathbf{7 a}$ ' with a ratio of isomers of $53 / 47$ (Table 1 , entry 2). In the case of phenylsulfonyl ethyl acetate $\mathbf{5 b}$ the base of choice was the 2,6-lutidine. Cycloaddition of sulfine $\mathbf{6 b}$ with 2,3-dimethyl-1,3-butadiene led to $7 \mathbf{b}$ as a mixture of two isomers in a 81/19 ratio and with 1,3-butadiene to cycloadduct $\mathbf{7 b}$ ' in a 57/43 ratio (Table 1, entries 3 and 4). Crystallization of $\mathbf{7 b}$ ' afforded a sample of pure minor isomer, from which a single crystal was isolated. The X-ray analysis showed a relative configuration in which the 
phenylsulfonyl group is located cis to the sulfinyl oxygen atom (Figure 2). The results obtained with phenylsulfonylacetyl oxazolidinone 5c were similar to those obtained with $\mathbf{5 a}$ and $\mathbf{5 b}$. Cycloadduct 7c resulting from 2,3-dimethyl-1,3-butadiene was obtained with a diastereomeric ratio of 87/13 and cycloadduct 7c' resulting from 1,3-butadiene with 67/33 dr (Table 1, entries 5 and 6). The assignment of the trans relative configuration for the major isomer of $7 \mathbf{b}$ ' let suppose that the major isomer obtained in the other similar cases (7a,a', 7b, and 7c, $\mathbf{7}$ ') also possesses the trans configuration. The differences observed in the cis/trans ratios of the cycloadducts resulting from the same sulfine (but different dienes) suggest sulfine isomerization, which could maybe occur under the reaction conditions via addition/elimination of a nucleophile (the tertiary amine used as the base or the $\mathrm{Cl}^{-}$ion).

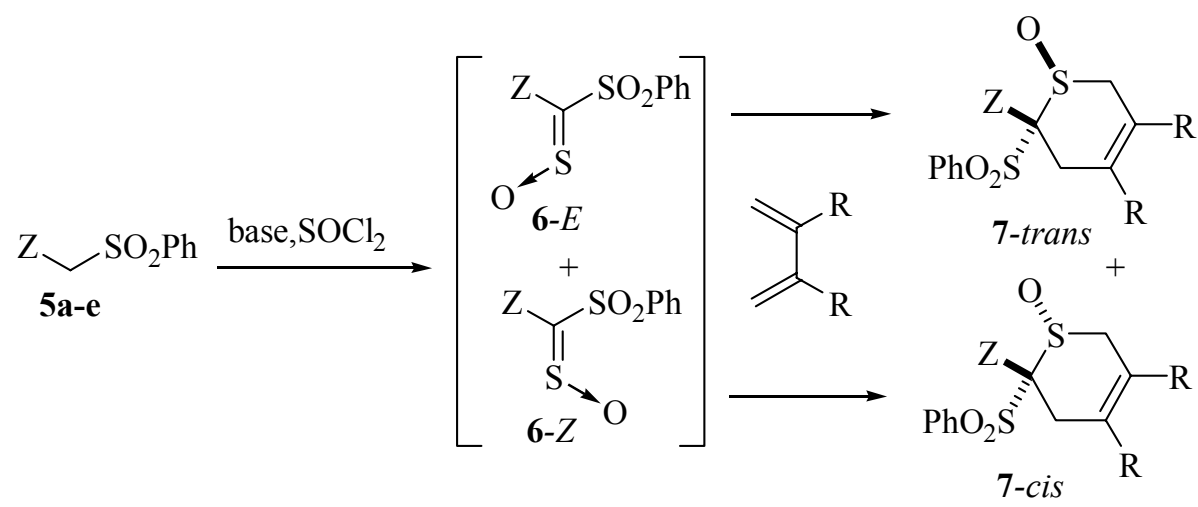

\section{Scheme 3}

Table 1

\begin{tabular}{ccccccc}
\hline Entry & Sulfone & $\mathrm{R}$ & Base & Cycloadduct & $\begin{array}{c}\mathrm{dr}^{\mathrm{a}} \\
\text { major/minor (\%) }\end{array}$ & $\begin{array}{c}\text { Isolated } \\
\text { yield (\%) }\end{array}$ \\
\hline 1 & $\mathbf{5 a}$ & $\mathrm{Me}$ & $\mathrm{NEt}_{3}$ & $\mathbf{7 a}$ & $67 / 33$ & 75 \\
2 & $\mathbf{5 a}$ & $\mathrm{H}$ & $\mathrm{NEt}_{3}$ & $\mathbf{7 a}$ & $53 / 47$ & 62 \\
3 & $\mathbf{5 b}$ & $\mathrm{Me}$ & $2,6-\mathbf{a l t i d i n e}$ & $\mathbf{7 b}$ & $81 / 19$ & 75 \\
4 & $\mathbf{5 b}$ & $\mathrm{H}$ & $2,6-$ lutidine & $\mathbf{7 b}$ & $57 / 43^{\mathrm{b}}$ & 67 \\
5 & $\mathbf{5 c}$ & $\mathrm{Me}$ & $\mathrm{NEt}_{3}$ & $\mathbf{7 c}$ & $87 / 13$ & 78 \\
6 & $\mathbf{5 c}$ & $\mathrm{H}$ & $\mathrm{NEt}_{3}$ & $\mathbf{7 c}$ & $67 / 33$ & 66 \\
7 & $\mathbf{5 d}$ & $\mathrm{Me}$ & $2,6-$ lutidine & $\mathbf{7 d}$ & $100 / 0^{\mathrm{b}}$ & 88 \\
8 & $\mathbf{5 e}$ & $\mathrm{Me}$ & $2,6-$ lutidine & $\mathbf{7 e}$ & $100 / 0$ & 74 \\
\hline
\end{tabular}

${ }^{\mathrm{a}}$ Diastereomeric ratio determinated by ${ }^{1} \mathrm{H}$ NMR; trans and cis isomers were not assigned. ${ }^{\mathrm{b}}$ Trans relative configuration was assigned to the major isomer. 
Then, we performed the reaction between the sulfine generated from methyl-2-pyridyl phenylsulfone 5d with the 2,3-dimethyl-1,3-butadiene. The expected cycloadduct 7d was obtained with a good isolated yield of $88 \%$ and as a single isomer (Table 1, entry 7). It was possible again to obtain a single crystal of this product and its analysis by X-ray diffraction showed a relative trans stereochemical arrangement between the phenylsulfonyl group and the sulfinyl oxygen atom (Figure 3). This supposes that the $E$ sulfine ${ }^{11}$ was selectively formed in situ. A similar result was obtained starting from the quinoline sulfone derivative $\mathbf{5 e}$, which led to cycloadduct $7 \mathbf{e}$ in $74 \%$ yield and as a single isomer, having very probably also trans configuration (Table 1 , entry 8 ).

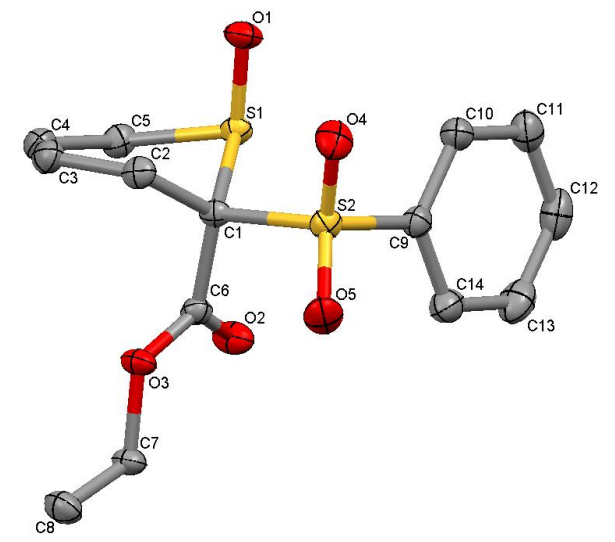

Figure 2. X-ray structure of the minor cis isomer of cycloadduct $\mathbf{7 b}$ '.

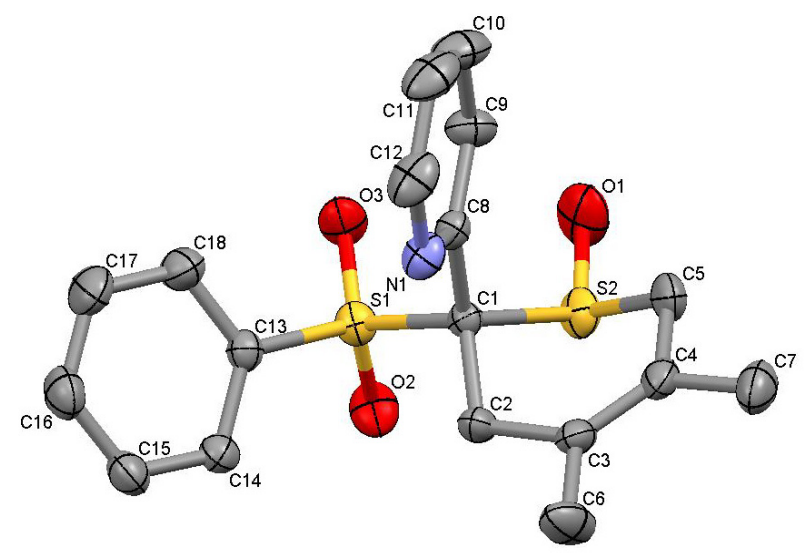

Figure 3. X-ray structure of the single trans isomer of cycloadduct 7d.

\section{Conclusions}

These results show that in the 5a-c sulfones series, when the sulfonyl group and the second substituent on the sulfine carbon have comparable electronwithdrawing effect, the sulfine is 
formed as a mixture of $Z$ and $E$ isomers, leading respectively to the corresponding cis and trans isomers of the cycloadduct, with low to moderate selectivities (53/47 to 87/13 ratio). For sulfones $\mathbf{5 d}$ and $\mathbf{5 e}$, due to the much stronger electronwithdrawing effect of the sulfonyl group compared to pyridyl or quinolyl substituent, only one isomer of the cycloadduct was obtained. Crystals of cycloadducts $\mathbf{7 b}$ ' (carboxylsulfonylsulfine with butadiene) and 7d (2-pyridyl sulfonylsulfine with 2,3-dimethylbutadiene) were isolated and analyzed by X-ray diffraction; the stereochemical arrangement was cis for the minor isomer of $\mathbf{7 b}$ ' and trans for the single isomer of 7d. Synthetic applications of the obtained cycloadducts are currently under examination in our laboratory.

\section{Experimental Section}

General. The solvents used in the reactions were purified using a PURESOLVTM apparatus developed by Innovative Technology Inc. Reactions were monitored by TLC using $0.25 \mathrm{~mm}$ thick Merck plates, silica gel 60 F254. Products were purified by flash column chromatography on silica gel Merck (40-63 $\mu \mathrm{m}, 60.08 \mathrm{~g} / \mathrm{mol})$. NMR spectra were recorded with a Bruker DRX $400 \mathrm{MHz}$ or a Bruker DRX $500 \mathrm{MHz}$ spectrometer in $\mathrm{CDCl}_{3} \cdot{ }^{13} \mathrm{C}$ and ${ }^{31} \mathrm{P} \mathrm{NMR}$ spectra were obtained with complete proton decoupling. The chemical shifts $(\delta)$ are given in parts per millions (ppm) relative to tetramethylsilane (TMS) for ${ }^{1} \mathrm{H}$ and ${ }^{13} \mathrm{C}$ nuclei, and to $\mathrm{H}_{3} \mathrm{PO}_{4}$ for ${ }^{31} \mathrm{P}$ nucleus. Conventional abbreviations are used: $\mathrm{s}=$ singlet, $\mathrm{d}=$ doublet, $\mathrm{t}=$ triplet, $\mathrm{q}=$ quartet, $\mathrm{qn}=$ quintet, $\mathrm{m}=$ multiplet, $\mathrm{br}=$ broad. Coupling constants $(J)$ are given in Hertz $(\mathrm{Hz})$. Mass spectra were obtained on a GC/MS Saturn 2000 spectrometer. High-resolution mass spectra (HRMS) were performed on Q-TOF Micro WATERS by electrospray ionisation (ESI). Infrared (IR) spectra were recorded with a Perkin Elmer 16 PC FT-IR spectrometer.

Sulfones 5a (RN: 206256-73-7), ${ }^{12}$ 5b (RN: 7605-30-3), ${ }^{5}$ 5d, ${ }^{10}$ and 5e (RN: 65492-27-5) ${ }^{10}$ have been prepared as previously described in the literature.

Synthesis of 3-(2-phenylsulfonylacetyl)oxazolidin-2-one (5c). A mixture of 3-(2chloroacetyl)oxazolidin-2-one (RN: 460313-68-2; $10 \mathrm{mmol}$ ), sodium benzenesulfinate (15 $\mathrm{mmol})$, and catalytic amount of tetrapropylammonium bromide ( $2 \mathrm{mmol})$, in acetonitrile (20 $\mathrm{mL}$ ), was refluxed for $12 \mathrm{~h}$. Then the solvent was removed under vacuum and the residue was dissolved in dichloromethane $(20 \mathrm{~mL})$, washed with brine $(20 \mathrm{~mL})$, dried $\left(\mathrm{MgSO}_{4}\right)$ and the solvent removed. The crude product was obtained in $56 \%$ as a white solid and was used without purification in the next step. ${ }^{1} \mathrm{H}$ NMR $\left(400.13 \mathrm{MHz}, \mathrm{CDCl}_{3}\right) \delta 7.90\left(\mathrm{~d}, J=7.6 \mathrm{~Hz}, 2 \mathrm{H}, \mathrm{H}^{\mathrm{ar}}\right), 7.62$ $\left(\mathrm{t}, J=6.0 \mathrm{~Hz}, 1 \mathrm{H}, \mathrm{H}^{\mathrm{ar}}\right), 7.52\left(\mathrm{~m}, 2 \mathrm{H}, \mathrm{H}^{\mathrm{ar}}\right), 4.87\left(\mathrm{~s}, 2 \mathrm{H}, \mathrm{CH}_{2} \mathrm{SO}_{2}\right), 4.35\left(\mathrm{t}, J=7.6 \mathrm{~Hz}, 2 \mathrm{H}, \mathrm{CH}_{2}-\mathrm{O}\right)$, $3.97\left(\mathrm{t}, J=7.6 \mathrm{~Hz}, 2 \mathrm{H}, \mathrm{CH}_{2} \mathrm{~N}\right) ;{ }^{13} \mathrm{C} \mathrm{NMR}\left(100.6 \mathrm{MHz}, \mathrm{CDCl}_{3}\right) \delta 161.2(\mathrm{C}=\mathrm{O}), 153.0(\mathrm{NC}=\mathrm{O})$, $139.3\left(\mathrm{C}^{\mathrm{ar}}\right), 134.3\left(\mathrm{CH}^{\mathrm{ar}}\right), 129.3\left(\mathrm{CH}^{\mathrm{ar}}\right), 128.5\left(\mathrm{CH}^{\mathrm{ar}}\right), 62.2\left(\mathrm{~s}, \mathrm{CH}_{2} \mathrm{O}\right), 59.5\left(\mathrm{CH}_{2} \mathrm{SO}_{2}\right), 42.6$ $\left(\mathrm{CH}_{2} \mathrm{~N}\right)$. HRMS Calcd for $\mathrm{C}_{11} \mathrm{H}_{12} \mathrm{NO}_{5} \mathrm{~S}[\mathrm{M}+\mathrm{H}]$ 270.0436. Found 270.0459. IR (neat): 2961, 
1766, 1699, 1448, 1398, 1368, 1326, 1211, 1154, 1123, 1102, 1054, 1024, 998, 972, 759, 728, $687 \mathrm{~cm}^{-1}$.

\section{General procedure for the thia-Diels-Alder reaction}

Under a nitrogen atmosphere a solution of sulfone 5 (1 mmol) and, as a base, 2,6-lutidine or triethylamine $(2.1 \mathrm{mmol})$ in THF $(5 \mathrm{~mL})$, was added dropwise to a cooled $\left(-78{ }^{\circ} \mathrm{C}\right)$ solution of thionyl chloride $(88 \mu \mathrm{L}, 1.2 \mathrm{mmol})$ and diene (2,3-dimethyl-1,3-butadiene or 1,3-butadiene, 5 to $10 \mathrm{mmol})$ in THF $(10 \mathrm{~mL})$. Once addition was complete, the temperature was allowed to rise slowly to room temperature and stirring was continued overnight. In the cases of substrates 5a-c, the crude reaction mixture was filtered, then the filtrate directly purified by chromatography on a silica gel column. In the cases of substrates $\mathbf{5} \mathbf{d}$ and $\mathbf{5 e}$, the reaction mixture was poured into a saturated aqueous solution of $\mathrm{NaHCO}_{3}(10 \mathrm{~mL})$, the organic layer separated, the aqueous layer extracted again with $\mathrm{CH}_{2} \mathrm{Cl}_{2}(20 \mathrm{~mL})$, and the combined organic layers were dried $\left(\mathrm{MgSO}_{4}\right)$ and filtered. Removal of the solvents in vacuum gave the cycloadduct 7 , which was purified by column chromatography (silica gel, ethyl acetate/pentane).

\section{2-Phenylsulfonyl-3,6-dihydro-4,5-dimethyl-1-oxo-2H-thiopyran-2-diisopropylphosphonate}

(7a). Prepared by the general procedure from diisopropyl 2-phenylsulfonylmethylphosphonate 5a and 2,3-dimethyl-1,3-butadiene, using triethylamine as a base. Flash chromatography (pentane/EtOAc, 1:1) afforded $336 \mathrm{mg}$ (75\% yield) of a mixture of trans and cis isomers (major/minor ratio: 67/33), as a brown oil.

Major diastereoisomer. ${ }^{31} \mathrm{P}$ NMR $\left(162.0 \mathrm{MHz}, \mathrm{CDCl}_{3}\right) \delta 9.5 .{ }^{1} \mathrm{H} \mathrm{NMR}\left(400.13 \mathrm{MHz}, \mathrm{CDCl}_{3}\right) \delta$ $8.13\left(\mathrm{~d}, J=8.4 \mathrm{~Hz}, 2 \mathrm{H}, \mathrm{H}^{\mathrm{ar}}\right), 7.61\left(\mathrm{t}, J=7.6 \mathrm{~Hz}, 1 \mathrm{H}, \mathrm{H}^{\mathrm{ar}}\right), 7.51\left(\mathrm{t}, J=8.4 \mathrm{~Hz}, 2 \mathrm{H}, \mathrm{H}^{\mathrm{ar}}\right), 4.93-4.85$ (m, 2H, 2xCH-O), 3.85 and 3.49 (AB system, $\left.J=15.7 \mathrm{~Hz}, 2 \mathrm{H}, \mathrm{SCH}_{2}\right), 2.98$ and $2.71(\mathrm{ABX}$ system, $J=16.6 \mathrm{~Hz}, 21.9 \mathrm{~Hz}$ and $\left.11.3 \mathrm{~Hz}, 2 \mathrm{H}, \mathrm{CH}_{2}\right), 1.72\left(\mathrm{~s}, 3 \mathrm{H}, \mathrm{CH}_{3}\right), 1.38\left(\mathrm{~s}, 3 \mathrm{H}, \mathrm{CH}_{3}\right), 1.38-$ $1.26\left(\mathrm{~m}, 12 \mathrm{H}, 4 \mathrm{xCH}_{3}\right) .{ }^{13} \mathrm{C} \mathrm{NMR}\left(100.6 \mathrm{MHz}, \mathrm{CDCl}_{3}\right) \delta 138.4\left(\mathrm{C}^{\mathrm{ar}}\right), 134.2\left(\mathrm{CH}^{\mathrm{ar}}\right), 131.3$ $\left(2 \mathrm{xCH}^{\mathrm{ar}}\right), 128.7(\mathrm{Cq}), 128.0\left(2 \mathrm{xCH}^{\mathrm{ar}}\right), 124.7(\mathrm{Cq}), 82.4(\mathrm{~d}, J=132.4 \mathrm{~Hz}, \mathrm{Cq}), 74.2-72.1(\mathrm{~m}$, $2 \mathrm{xCH}), 54.5\left(\mathrm{CH}_{2}\right), 30.6\left(\mathrm{CH}_{2}\right), 24.0-23.3\left(\mathrm{~m}, 4 \mathrm{xCH}_{3}\right), 19.9\left(\mathrm{CH}_{3}\right), 19.5\left(\mathrm{CH}_{3}\right)$.

Minor diastereoisomer. ${ }^{31} \mathrm{P} \mathrm{NMR}\left(162.0 \mathrm{MHz}, \mathrm{CDCl}_{3}\right) \delta 9.4 .{ }^{1} \mathrm{H} \mathrm{NMR}\left(400.13 \mathrm{MHz}, \mathrm{CDCl}_{3}\right) \delta$ $8.09\left(\mathrm{~d}, J=8.4 \mathrm{~Hz}, 2 \mathrm{H}, \mathrm{H}^{\mathrm{ar}}\right), 7.68\left(\mathrm{t}, J=7.6 \mathrm{~Hz}, 1 \mathrm{H}, \mathrm{H}^{\mathrm{ar}}\right), 7.56\left(\mathrm{t}, J=8.4 \mathrm{~Hz}, 2 \mathrm{H}, \mathrm{H}^{\mathrm{ar}}\right), 4.89-4.81$ $(\mathrm{m}, 2 \mathrm{H}, 2 \mathrm{xCH}-\mathrm{O}), 4.04$ and $3.49\left(\mathrm{AB}\right.$ system, $\left.J=14.0 \mathrm{~Hz}, 2 \mathrm{H}, \mathrm{SCH}_{2}\right), 3.20$ and $2.80(\mathrm{AB}$ system, $\left.J=15.2 \mathrm{~Hz}, 2 \mathrm{H}, \mathrm{CH}_{2}\right), 1.82\left(\mathrm{~s}, 6 \mathrm{H}, 2 \mathrm{xCH}_{3}\right), 1.34-1.17\left(\mathrm{~m}, 12 \mathrm{H}, 4 \mathrm{xCH}_{3}\right) .{ }^{13} \mathrm{C} \mathrm{NMR}$ $\left(100.6 \mathrm{MHz}, \mathrm{CDCl}_{3}\right) \delta 137.1\left(\mathrm{C}^{\mathrm{ar}}\right), 134.4\left(\mathrm{CH}^{\mathrm{ar}}\right), 131.6\left(2 \mathrm{xCH}^{\mathrm{ar}}\right), 129.1(\mathrm{Cq}), 128.4\left(2 \mathrm{xCH}^{\mathrm{ar}}\right)$, $120.3(\mathrm{Cq}), 86.5(\mathrm{~d}, J=135.2 \mathrm{~Hz}, \mathrm{Cq}), 73.4-73.0(\mathrm{~m}, 2 \mathrm{xCH}), 53.6\left(\mathrm{CH}_{2}\right), 33.6\left(\mathrm{CH}_{2}\right), 24.2-23.2$ $\left(\mathrm{m}, 4 \mathrm{xCH}_{3}\right), 20.1\left(\mathrm{CH}_{3}\right), 19.7\left(\mathrm{CH}_{3}\right) . \mathrm{MSMS}:[\mathrm{M}+\mathrm{H}]: 449$ (27), 407 (100), 365 (29), 307 (13), 267 (21), 183 (14), 141 (10).HRMS Calcd for $\mathrm{C}_{19} \mathrm{H}_{30} \mathrm{O}_{6} \mathrm{PS}_{2}[\mathrm{M}+\mathrm{H}]$ 449.1221. Found 449.1244. IR (neat): 2980, 1145, 1382, 1310, 1255, 1144, 1076, 981, 881, 811, 754, $680 \mathrm{~cm}^{-1}$.

2-Phenylsulfonyl-3,6-dihydro-1-oxo-2H-thiopyran-2-diisopropylphosphonate (7a').

Prepared by the general procedure from diisopropyl 2-phenylsulfonylmethylphosphonate 5a and 1,3-butadiene, using triethylamine as a base. Flash chromatography (pentane/EtOAc, 2:8) 
afforded $260 \mathrm{mg}$ (62\% yield) of a mixture of trans and cis isomers (major/minor ratio: 53/47), as a brown oil.

Major diastereoisomer. ${ }^{31} \mathrm{P} \mathrm{NMR}\left(162.0 \mathrm{MHz}, \mathrm{CDCl}_{3}\right) \delta 8.7 .{ }^{1} \mathrm{H} \mathrm{NMR}\left(400.13 \mathrm{MHz}, \mathrm{CDCl}_{3}\right) \delta$ $8.05\left(\mathrm{~d}, J=7.3 \mathrm{~Hz}, 2 \mathrm{H}, \mathrm{H}^{\mathrm{ar}}\right), 7.62\left(\mathrm{t}, J=7.2 \mathrm{~Hz}, 1 \mathrm{H}, \mathrm{H}^{\mathrm{ar}}\right), 7.50\left(\mathrm{t}, J=8.4 \mathrm{~Hz}, 2 \mathrm{H}, \mathrm{H}^{\mathrm{ar}}\right), 5.90-5.82$ (m, 1H, CH), 5.70-5.62 (m, 1H, CH), 4.96-4.80 (m, 2H, 2xCH-O), 4.00 and 3.52 (ABX system, $J$ $=17.2 \mathrm{~Hz}$ and $\left.4.9 \mathrm{~Hz}, 2 \mathrm{H}, \mathrm{SCH}_{2}\right), 3.06-2.81\left(\mathrm{~m}, 2 \mathrm{H}, \mathrm{CH}_{2}\right), 1.39-1.18\left(\mathrm{~m}, 12 \mathrm{H}, 4 \mathrm{xCH}_{3}\right) .{ }^{13} \mathrm{C}$ NMR $\left(100.6 \mathrm{MHz}, \mathrm{CDCl}_{3}\right) \delta 137.8\left(\mathrm{C}^{\mathrm{ar}}\right), 134.4\left(\mathrm{CH}^{\mathrm{ar}}\right), 131.6\left(2 \mathrm{xCH}^{\mathrm{ar}}\right), 128.3\left(2 \mathrm{xCH}^{\mathrm{ar}}\right), 127.3$ $(\mathrm{CH}), 117.9(\mathrm{CH}), 84.7(\mathrm{~d}, J=133.2 \mathrm{~Hz}, \mathrm{Cq}), 74.0-73.7(\mathrm{~m}, 2 \mathrm{xCH}), 50.0\left(\mathrm{CH}_{2}\right), 26.9\left(\mathrm{CH}_{2}\right)$, 24.4-23.7 (m, $\left.4 \mathrm{xCH}_{3}\right)$.

Minor diastereoisomer. ${ }^{31} \mathrm{P} \mathrm{NMR}\left(162.0 \mathrm{MHz}, \mathrm{CDCl}_{3}\right) \delta 9.3 .{ }^{1} \mathrm{H} \mathrm{NMR}\left(400.13 \mathrm{MHz}, \mathrm{CDCl}_{3}\right) \delta$ $8.09\left(\mathrm{~d}, J=7.3 \mathrm{~Hz}, 2 \mathrm{H}, 2 \mathrm{H}^{\mathrm{ar}}\right), 7.68\left(\mathrm{t}, J=7.4 \mathrm{~Hz}, 1 \mathrm{H}, \mathrm{H}^{\mathrm{ar}}\right), 7.55\left(\mathrm{t}, J=8.0 \mathrm{~Hz}, 2 \mathrm{H}, \mathrm{H}^{\mathrm{ar}}\right), 5.94(\mathrm{dt}$, $J=9.9 \mathrm{~Hz}$ and $4.8 \mathrm{~Hz}, 1 \mathrm{H}, \mathrm{CH}), 5.78(\mathrm{dt}, J=10.2 \mathrm{~Hz}$ and $5.2 \mathrm{~Hz}, 1 \mathrm{H}, \mathrm{CH}), 4.96-4.82(\mathrm{~m}, 2 \mathrm{H}$, 2xCH-O), 4.05 and 3.81 (ABX system, $J=14.7 \mathrm{~Hz}, 6.0 \mathrm{~Hz}$ and $5.0 \mathrm{~Hz}, 2 \mathrm{H}, \mathrm{SCH}_{2}$ ), 3.24 and 2.92 (ABX system, $J=13.0 \mathrm{~Hz}, 5.4 \mathrm{~Hz}$ and $\left.4.7 \mathrm{~Hz}, 2 \mathrm{H}, \mathrm{CH}_{2}\right), 1.39-1.20\left(\mathrm{~m}, 12 \mathrm{H}, 4 \mathrm{xCH}_{3}\right) .{ }^{13} \mathrm{C}$ NMR (100.6 MHz, $\left.\mathrm{CDCl}_{3}\right) \delta 137.8\left(\mathrm{C}^{\mathrm{ar}}\right), 134.6\left(\mathrm{CH}^{\mathrm{ar}}\right), 131.5\left(2 \mathrm{xCH}^{\mathrm{ar}}\right), 128.5\left(2 \mathrm{xCH}^{\mathrm{ar}}\right), 127.3$ $(\mathrm{CH}), 119.9(\mathrm{CH}), 84.7(\mathrm{~d}, J=133.2 \mathrm{~Hz}, \mathrm{Cq}), 74.0-73.7(\mathrm{~m}, 2 \mathrm{xCH}), 48.7\left(\mathrm{CH}_{2}\right), 26.9\left(\mathrm{CH}_{2}\right)$, 24.4-23.7 (m, 4xCH$)$. HRMS Calcd for $\mathrm{C}_{17} \mathrm{H}_{26} \mathrm{O}_{6} \mathrm{PS}_{2}[\mathrm{M}+\mathrm{H}]$ 421.0908. Found 421.0898. IR (neat): 2981, 1148, 1386, 1312, 1255, 1146, 1078, 981, 889, 813, 754, $686 \mathrm{~cm}^{-1}$.

2-Phenylsulfonyl-3,6-dihydro-4,5-dimethyl-1-oxo-2H-thiopyran-2-ethylcarboxylate (7b). Prepared by the general procedure from ethyl 2-methylsulfonylacetate $\mathbf{5 b}$ and 2,3-dimethyl-1,3butadiene, using 2,6-lutidine as a base. Flash chromatography (pentane/EtOAc, 1:1) afforded 267 $\mathrm{mg}$ (75\% yield) of a mixture of trans and cis isomers (major/minor ratio: 81/19), as a brown oil. As this compound was already described, ${ }^{5}$ only some spectral and physical data of the major isomer are given here.

Major diastereoisomer. ${ }^{1} \mathrm{H}$ NMR $\left(400.13 \mathrm{MHz}, \mathrm{CDCl}_{3}\right) \delta 7.92(\mathrm{~d}, J=8.4 \mathrm{~Hz}, 2 \mathrm{H}, \mathrm{H}$ ) $7.69(\mathrm{t}$, $\left.J=8.4 \mathrm{~Hz}, 1 \mathrm{H}, \mathrm{H}^{\mathrm{ar}}\right) 7.56\left(\mathrm{t}, J=8.4 \mathrm{~Hz}, 2 \mathrm{H}, \mathrm{H}^{\mathrm{ar}}\right), 4.12-3.95\left(\mathrm{~m}, 2 \mathrm{H}, \mathrm{O}-\mathrm{CH}_{2}\right), 3.76$ and $3.52(\mathrm{AB}$ system, $\left.2 \mathrm{H}, J=16.5 \mathrm{~Hz}, 2 \mathrm{H}, \mathrm{SCH}_{2}\right), 3.17$ and 2.77 (AB system, $\left.J=17.6 \mathrm{~Hz}, 2 \mathrm{H}, \mathrm{CH}_{2}\right), 1.75$ (s, $\left.3 \mathrm{H}, \mathrm{CH}_{3}\right), 1.72\left(\mathrm{~s}, 3 \mathrm{H}, \mathrm{CH}_{3}\right), 1.11\left(\mathrm{t}, J=7.0 \mathrm{~Hz}, 3 \mathrm{H}, \mathrm{CH}_{3}\right)$. MSMS: [M+H] 357 (57), 215 (100), 197 (97), 169 (13), 165 (38). HRMS Calcd for $\mathrm{C}_{16} \mathrm{H}_{21} \mathrm{O}_{5} \mathrm{~S}_{2}[\mathrm{M}+\mathrm{H}]$ 357.0830. Found 357.0827. IR (neat): 2985, 1729, 1448, 1393, 1325, 1225, 1147, 1059, 1022, 912, 854, 800, 761, 716, 687.

2-Phenylsulfonyl-3,6-dihydro-1-oxo-2H-thiopyran-2-ethylcarboxylate (7b'). Prepared by the general procedure from ethyl 2-methylsulfonylacetate $\mathbf{5 b}$ and 1,3-butadiene, using 2,6-lutidine as a base. Flash chromatography (pentane/EtOAc, 4:6) afforded $220 \mathrm{mg}$ (67\% yield) of a mixture of trans and cis isomers (major/minor ratio: 57/43), as a brown oil. The product (100 $\mathrm{mg}$ ) was dissolved in a mixture of pentane/EtOAc 8:2 (1 mL) and by a long standing at the room temperature (about two weeks on the bench), crystals of minor $\mathbf{7 b}$ ' were separated from the solution which contained both isomers in the mixture.

Major diastereoisomer. Aspect: brown oil. ${ }^{1} \mathrm{H}$ NMR $\left(400.13 \mathrm{MHz}, \mathrm{CDCl}_{3}\right) \delta 7.92(\mathrm{~d}, J=8.4$ $\left.\mathrm{Hz}, 2 \mathrm{H}, \mathrm{H}^{\mathrm{ar}}\right), 7.71\left(\mathrm{t}, J=7.6 \mathrm{~Hz}, 1 \mathrm{H}, \mathrm{H}^{\mathrm{ar}}\right), 7.61-7.26\left(\mathrm{~m}, 2 \mathrm{H}, \mathrm{H}^{\mathrm{ar}}\right), 6.03-5.93(\mathrm{~m}, 1 \mathrm{H}, \mathrm{CH}), 5.77-$ $5.65(\mathrm{~m}, 1 \mathrm{H}, \mathrm{CH}), 4.27-4.17\left(\mathrm{~m}, 2 \mathrm{H}, \mathrm{OCH}_{2}\right), 4.03-3.93$ and $3.65-3.58\left(\mathrm{~m}, 2 \mathrm{H}, \mathrm{SCH}_{2}\right), 3.21-3.12$ 
and 2.99-2.96 (m, 2H, CH$), 1.11\left(\mathrm{t}, J=7.2 \mathrm{~Hz}, 3 \mathrm{H}, \mathrm{CH}_{3}\right) .{ }^{13} \mathrm{C} \mathrm{NMR}\left(100.6 \mathrm{MHz}, \mathrm{CDCl}_{3}\right) \delta$ $163.8(\mathrm{C}=\mathrm{O}), 136.8\left(\mathrm{C}^{\mathrm{ar}}\right), 135.2\left(\mathrm{CH}^{\mathrm{ar}}\right), 130.1\left(2 \mathrm{xCH}^{\mathrm{ar}}\right), 129.1\left(2 \mathrm{xCH}^{\mathrm{ar}}\right), 125.3(\mathrm{CH}), 116.7$ $(\mathrm{CH}), 85.5(\mathrm{Cq}), 63.7\left(\mathrm{O}-\mathrm{CH}_{2}\right), 47.6\left(\mathrm{CH}_{2}\right), 24.5\left(\mathrm{CH}_{2}\right), 13.6\left(\mathrm{CH}_{3}\right)$.

Minor diastereoisomer. Aspect: brown crystals; mp 135-136 ${ }^{\circ} \mathrm{C}$. ${ }^{1} \mathrm{H}$ NMR $(400.13 \mathrm{MHz}$, $\left.\mathrm{CDCl}_{3}\right) \delta 8.00\left(\mathrm{~d}, J=8.4 \mathrm{~Hz}, 2 \mathrm{H}, \mathrm{H}^{\mathrm{ar}}\right), 7.74\left(\mathrm{t}, J=7.4 \mathrm{~Hz}, 1 \mathrm{H}, \mathrm{H}^{\mathrm{ar}}\right), 7.61\left(\mathrm{t}, J=8.2 \mathrm{~Hz}, 2 \mathrm{H}, \mathrm{H}^{\mathrm{ar}}\right)$, 6,02-5.98 (m, 1H, CH), 5.57-5.53 (m, 1H, CH), 4.26-4.20 (m, 2H, $\left.\mathrm{OCH}_{2}\right), 3.35-3.32(\mathrm{~m}, 2 \mathrm{H}$, $\left.\mathrm{SCH}_{2}\right), 3.01-2.97\left(\mathrm{~m}, 2 \mathrm{H}, \mathrm{CH}_{2}\right), 1.27\left(\mathrm{t}, J=7.1 \mathrm{~Hz}, 3 \mathrm{H}, \mathrm{CH}_{3}\right) .{ }^{13} \mathrm{C} \mathrm{NMR}\left(100.6 \mathrm{MHz}, \mathrm{CDCl}_{3}\right) \delta$ $163.5(\mathrm{C}=\mathrm{O}), 136.8\left(\mathrm{C}^{\mathrm{ar}}\right), 135.2\left(\mathrm{CH}^{\mathrm{ar}}\right), 130.7\left(2 \mathrm{xCH}^{\mathrm{ar}}\right), 129.1\left(2 \mathrm{xCH}^{\mathrm{ar}}\right), 126.4(\mathrm{CH}), 115.1$ $(\mathrm{CH}), 86.1(\mathrm{Cq}), 63.6\left(\mathrm{O}-\mathrm{CH}_{2}\right), 47.4\left(\mathrm{CH}_{2}\right), 20.8\left(\mathrm{CH}_{2}\right), 13.7\left(\mathrm{CH}_{3}\right) . \mathrm{MSMS}$ : [M+Na] $351(50)$, $[\mathrm{M}+\mathrm{H}] 329$ (100), 187 (41), 186 (55). HRMS Calcd for $\mathrm{C}_{14} \mathrm{H}_{17} \mathrm{O}_{5} \mathrm{~S}_{2}[\mathrm{M}+\mathrm{H}]$ 329.0517. Found 329.0527. IR (neat): 2985, 1729, 1448, 1393, 1325, 1225, 1147, 1059, 1022, 912, 854, 800, 761, $716,687$.

Crystal data of minor 7b'. Bruker Kappa APEXII CCD diffractometer $\left(\mathrm{Mo}_{\mathrm{K} \alpha} \lambda=0.71073 \AA\right.$; graphite monochromator; $T=100(2) \mathrm{K})$. Formula $\mathrm{C}_{14} \mathrm{H}_{16} \mathrm{O}_{5} \mathrm{~S}_{2}$, formula weight 328.39 , crystal system monoclinic, space group $P 2_{1}$, crystal dimensions $0.39 \times 0.26 \times 0.11 \mathrm{~mm}^{3}, a=8.5112(2)$, $b=7.5858(2), c=11.4014(2) \AA, \beta=101.299(1)^{\circ}, V=721.86(3) \AA^{3}, Z=2, \rho_{\text {calcd }}=1.511 \mathrm{Mgm}^{-3}$, $\mu=0.39 \mathrm{~mm}^{-1}, 2 \theta_{\max }=74.00^{\circ}, 25175$ measured reflections, 5279 independent reflections $\left(R_{\text {int }}=\right.$ $0.025), R 1[I>2 \sigma(I)]=0.023, w R 2[I>2 \sigma(I)]=0.058, \mathrm{GOF}=1.04,254$ parameters, final difference map within 0.31 and $-0.23 \mathrm{e}^{-3}$. The structure was solved using direct methods and refined by full-matrix least-squares analysis on $F^{2}$. Numerical absorption corrections were applied (G. M. Sheldrick SHELXTL, Bruker ACS Inc.: Madison, WI, 2008). CCDC 784819 contains the supplementary crystallographic data for this paper. These data can be obtained free of charge from The Cambridge Crystallographic Data Center via www.ccdc.cam.ac.uk/data_request/cif. Selected bond lengths $(\AA)$ and angles (deg): S1-O1 1.4954(8), S1-C1 1.8625(8), S2-O4 1.4390(9), S2-O5 1.4396(8), S2-C1 1.8508(9), C4-C3 1.3307(15), S1-C1-S2 108.21(4), S1-C1C2 109.41(7), C3-C4-C5 126.19(9), C2-C3-C4 125.20(9).

\section{2-Phenylsulfonyl-3,6-dihydro-4,5-dimethyl-1-oxo-2H-thiopyran-2-(acetyloxazolidin-2-one)}

(7c). Prepared by the general procedure from sulfone 5c and 2,3-dimethyl-1,3-butadiene, using triethylamine as a base. Flash chromatography (pentane/EtOAc, 1:1) afforded $309 \mathrm{mg}$ (78\% yield) of a mixture of trans and cis isomers (major/minor ratio: 87/13), as a brown oil.

Major diastereoisomer. Aspect: brown paste; $\mathrm{Rf}$ (cyclohexane/EtOAc: $1 / 1)=0.15$.

${ }^{1} \mathrm{H}$ NMR $\left(500.13 \mathrm{MHz}, \mathrm{CDCl}_{3}\right) \delta 7.88\left(\mathrm{~d}, J=7.4 \mathrm{~Hz}, 2 \mathrm{H}, \mathrm{H}^{\mathrm{ar}}\right), 7.69\left(\mathrm{t}, J=7.6 \mathrm{~Hz}, 1 \mathrm{H}, \mathrm{H}^{\mathrm{ar}}\right), 7.56$ $\left(\mathrm{t}, J=8.2 \mathrm{~Hz}, 2 \mathrm{H}, \mathrm{H}^{\mathrm{ar}}\right), 4.49$ (t, $\left.J=7.8 \mathrm{~Hz}, 2 \mathrm{H}, \mathrm{CH}_{2} \mathrm{O}\right), 4.19\left(\mathrm{t}, J=7.8 \mathrm{~Hz}, 2 \mathrm{H}, \mathrm{CH}_{2} \mathrm{~N}\right), 4.06$ and 3.32 (AB system, $J=17.7 \mathrm{~Hz}, 2 \mathrm{H}, \mathrm{SCH}_{2}$ ), 3.17 and 2.85 (AB system, $J=18.3 \mathrm{~Hz}, 2 \mathrm{H}, \mathrm{CH}_{2}$ ), $1.67\left(\mathrm{~s}, 3 \mathrm{H}, \mathrm{CH}_{3}\right), 1.63\left(\mathrm{~s}, 3 \mathrm{H}, \mathrm{CH}_{3}\right) .{ }^{13} \mathrm{C} \mathrm{NMR}\left(100.6 \mathrm{MHz}, \mathrm{CDCl}_{3}\right) \delta 163.8(\mathrm{C}=\mathrm{O}), 151.7$ $(\mathrm{C}=\mathrm{O}), 138.0\left(\mathrm{C}^{\mathrm{ar}}\right), 134.5\left(\mathrm{CH}^{\mathrm{ar}}\right), 131.9\left(\mathrm{CH}^{\mathrm{ar}}\right), 130.0\left(\mathrm{CH}^{\mathrm{ar}}\right), 128.6\left(\mathrm{CH}^{\mathrm{ar}}\right), 124.8(\mathrm{Cq}), 118.6$ $(\mathrm{Cq}), 89.6(\mathrm{Cq}), 62.7\left(\mathrm{O}-\mathrm{CH}_{2}\right), 52.2\left(\mathrm{CH}_{2}\right), 45.0\left(\mathrm{~N}-\mathrm{CH}_{2}\right), 30.9\left(\mathrm{CH}_{2}\right), 19.5\left(\mathrm{CH}_{3}\right), 19.3\left(\mathrm{CH}_{3}\right)$.

Minor diastereoisomer. Aspect: brown oil; $\mathrm{Rf}($ cyclohexane/EtOAc: $1 / 1)=0.54$.

${ }^{1} \mathrm{H}$ NMR $\left(500.13 \mathrm{MHz}, \mathrm{CDCl}_{3}\right) \delta 7.90\left(\mathrm{~d}, J=8.5 \mathrm{~Hz}, 2 \mathrm{H}, \mathrm{H}^{\mathrm{ar}}\right), 7.68\left(\mathrm{t}, J=7.5 \mathrm{~Hz}, 1 \mathrm{H}, \mathrm{H}^{\mathrm{ar}}\right), 7.56$ $\left(\mathrm{t}, J=8.0 \mathrm{~Hz}, 2 \mathrm{H}, \mathrm{H}^{\mathrm{ar}}\right), 4.62-4.35\left(\mathrm{~m}, 2 \mathrm{H}, \mathrm{CH}_{2} \mathrm{O}\right), 4.43-4.24\left(\mathrm{~m}, 2 \mathrm{H}, \mathrm{CH}_{2} \mathrm{~N}\right), 3.12$ and $2.82(\mathrm{AB}$ 
system, $\left.J=14.2 \mathrm{~Hz}, 2 \mathrm{H}, \mathrm{SCH}_{2}\right), 3.09$ and 2.45 (AB system, $\left.J=14.2 \mathrm{~Hz}, 2 \mathrm{H}, \mathrm{CH}_{2}\right), 1.76(\mathrm{~s}, 3 \mathrm{H}$, $\left.\mathrm{CH}_{3}\right), 1.72\left(\mathrm{~s}, 3 \mathrm{H}, \mathrm{CH}_{3}\right) .{ }^{13} \mathrm{C} \mathrm{NMR}\left(100.6 \mathrm{MHz}, \mathrm{CDCl}_{3}\right) \delta 164.6(\mathrm{C}=\mathrm{O}), 152.4(\mathrm{C}=\mathrm{O}), 135.5$ $\left(\mathrm{C}^{\mathrm{ar}}\right), 134.5\left(\mathrm{CH}^{\mathrm{ar}}\right), 130.5\left(\mathrm{CH}^{\mathrm{ar}}\right), 129.5\left(\mathrm{CH}^{\mathrm{ar}}\right), 128.8\left(\mathrm{CH}^{\mathrm{ar}}\right), 128.1(\mathrm{Cq}), 125.5(\mathrm{Cq}), 83.8(\mathrm{Cq})$, $62.5\left(\mathrm{O}-\mathrm{CH}_{2}\right), 45.6\left(\mathrm{~N}-\mathrm{CH}_{2}\right), 37.9\left(\mathrm{CH}_{2}\right), 32.7\left(\mathrm{CH}_{2}\right), 19.2\left(\mathrm{CH}_{3}\right), 18.4\left(\mathrm{CH}_{3}\right)$. HRMS Calcd for $\mathrm{C}_{17} \mathrm{H}_{20} \mathrm{NO}_{6} \mathrm{~S}_{2}[\mathrm{M}+\mathrm{H}]$ 398.0732. Found 398.07245. IR (neat): 2915, 1784, 1673, 1582, 1476, $1447,1438,1385,1361,1308,1197,1147,1120,1040,998,778,753,720,688 \mathrm{~cm}^{-1}$.

2-Phenylsulfonyl-3,6-dihydro-1-oxo-2H-thiopyran-2-(acetyloxazolidin-2-one) (7c'). Prepared by the general procedure from sulfone 5c and 1,3-butadiene, using triethylamine as a base. Flash chromatography (pentane/EtOAc, 2:8) afforded $243 \mathrm{mg}$ (66\% yield) of a mixture of trans and cis isomers (major/minor ratio: 67/33), as a brown oil.

Major diastereoisomer. Aspect: brown oil; Rf (cyclohexane/EtOAc: $2 / 8$ ) $=0.72 .{ }^{1} \mathrm{H}$ NMR $\left(500.13 \mathrm{MHz}, \mathrm{CDCl}_{3}\right) \delta 7.95\left(\mathrm{~d}, J=8.3 \mathrm{~Hz}, 2 \mathrm{H}, \mathrm{H}^{\mathrm{ar}}\right), 7.68\left(\mathrm{t}, J=7.5 \mathrm{~Hz}, 1 \mathrm{H}, \mathrm{H}^{\mathrm{ar}}\right), 7.56(\mathrm{t}, J=$ $\left.7.5 \mathrm{~Hz}, 2 \mathrm{H}, \mathrm{H}^{\mathrm{ar}}\right), 6.10-5.95(\mathrm{~m}, 1 \mathrm{H}, \mathrm{CH}), 5.89-5.85(\mathrm{~m}, 1 \mathrm{H}, \mathrm{CH}), 4.56-4.36\left(\mathrm{~m}, 2 \mathrm{H}, \mathrm{CH}_{2} \mathrm{O}\right)$, 4.42-4.27 (m, 2H, $\left.\mathrm{CH}_{2} \mathrm{~N}\right), 4.20-4.03$ and 3.27-3.13 (m, AB system, $\left.2 \mathrm{H}, \mathrm{SCH}_{2}\right), 3.27$ and 2.71 (AB system, $\left.J=14.7 \mathrm{~Hz}, 2 \mathrm{H}, \mathrm{CH}_{2}\right) .{ }^{13} \mathrm{C} \mathrm{NMR}\left(100.6 \mathrm{MHz}, \mathrm{CDCl}_{3}\right) \delta 165.0(\mathrm{C}=\mathrm{O}), 152.2$ $(\mathrm{C}=\mathrm{O}), 136.1\left(\mathrm{C}^{\mathrm{ar}}\right), 134.5\left(\mathrm{CH}^{\mathrm{ar}}\right), 130.9\left(2 \mathrm{xCH}^{\mathrm{ar}}\right), 128.4\left(2 \mathrm{xCH}^{\mathrm{ar}}\right), 126.3(\mathrm{CH}), 124.7(\mathrm{CH}), 80.4$ $(\mathrm{Cq}), 62.6\left(\mathrm{O}-\mathrm{CH}_{2}\right), 45.7\left(\mathrm{~N}-\mathrm{CH}_{2}\right), 30.1\left(\mathrm{CH}_{2}\right), 26.5\left(\mathrm{CH}_{2}\right)$.

Minor diastereoisomer. Aspect: brown oil; $\mathrm{Rf}$ (cyclohexane/EtOAc: $1 / 1)=0.32$. ${ }^{1} \mathrm{H}$ NMR $\left(500.13 \mathrm{MHz}, \mathrm{CDCl}_{3}\right) \delta 8.10\left(\mathrm{~d}, J=8.5 \mathrm{~Hz}, 2 \mathrm{H}, \mathrm{H}^{\mathrm{ar}}\right), 7.68\left(\mathrm{t}, J=7.5 \mathrm{~Hz}, 1 \mathrm{H}, \mathrm{H}^{\mathrm{ar}}\right), 7.56(\mathrm{t}, J=$ $\left.8.0 \mathrm{~Hz}, 2 \mathrm{H}, \mathrm{H}^{\mathrm{ar}}\right), 5.91-5.84(\mathrm{~m}, 1 \mathrm{H}, \mathrm{CH}), 5.64-5.59(\mathrm{~m}, 1 \mathrm{H}, \mathrm{CH}), 5.52-5.39\left(\mathrm{~m}, 2 \mathrm{H}, \mathrm{CH}_{2} \mathrm{O}\right)$, 4.23-3.95 (m, 2H, $\mathrm{CH}_{2} \mathrm{~N}$ ), 3.78 and 3.24 (ABX system, $J=17.9 \mathrm{~Hz}, 7.6 \mathrm{~Hz}$ and $2.5 \mathrm{~Hz}, 2 \mathrm{H}$, $\mathrm{SCH}_{2}$ ), 3.55 and 3.19 (ABX system, $J=18.4 \mathrm{~Hz}, 2.7 \mathrm{~Hz}$ and $\left.2.0 \mathrm{~Hz}, 2 \mathrm{H}, \mathrm{CH}_{2}\right) .{ }^{13} \mathrm{C} \mathrm{NMR}(100.6$ $\left.\mathrm{MHz}, \mathrm{CDCl}_{3}\right) \delta 164.6(\mathrm{C}=\mathrm{O}), 151.3(\mathrm{C}=\mathrm{O}), 135.0\left(\mathrm{C}^{\mathrm{ar}}\right), 132.7\left(\mathrm{CH}^{\mathrm{ar}}\right), 131.9\left(\mathrm{CH}^{\mathrm{ar}}\right), 130.9$ $\left(\mathrm{CH}^{\mathrm{ar}}\right), 128.8\left(\mathrm{CH}^{\mathrm{ar}}\right), 128.4\left(\mathrm{CH}^{\mathrm{ar}}\right), 125.9(\mathrm{CH}), 119.1(\mathrm{CH}), 90.6(\mathrm{Cq}), 62.6\left(\mathrm{O}-\mathrm{CH}_{2}\right), 59.0$ $\left(\mathrm{CH}_{2}\right), 50.1\left(\mathrm{CH}_{2}\right)$. HRMS Calcd for $\mathrm{C}_{15} \mathrm{H}_{16} \mathrm{NO}_{6} \mathrm{~S}_{2}[\mathrm{M}+\mathrm{H}]$ 370.0419. Found 370.0424. IR (neat): 3063, 2981, 2924, 1778, 1682, 1583, 1476, 1447, 1385, 1362, 1308, 1217, 1142, 1079, 1038, $998,982,911,754,728,717,686 \mathrm{~cm}^{-1}$.

\section{2-Phenylsulfonyl-3,6-dihydro-4,5-dimethyl-1-oxo-2 $\mathrm{H}$-thiopyran-2-(2-pyridine)}

(7d). Prepared by the general procedure from 2-phenylsulfonylmethylpyridine 5d and 2,3-dimethyl1,3-butadiene, using 2,6-lutidine as a base. Flash chromatography (pentane/EtOAc, 2:1 to 1:2) afforded $0.319 \mathrm{~g}$ ( $88 \%$ yield) of a white crystalline solid, $\mathrm{mp} 151{ }^{\circ} \mathrm{C}$. The product $(100 \mathrm{mg})$ was dissolved in a mixture of pentane/EtOAc 6:4 $(1 \mathrm{~mL})$ and by standing at the room temperature, on the bench, about three days, crystals of $7 \mathbf{d}$ were separated from the solution.

${ }^{1} \mathrm{H}$ NMR (400.13 MHz, $\left.\mathrm{CDCl}_{3}\right) \delta 8.42(\mathrm{~m}, 1 \mathrm{H}), 7.93(\mathrm{~m}, 1 \mathrm{H}), 7.51(\mathrm{~m}, 4 \mathrm{H}), 7.31(\mathrm{~m}, 2 \mathrm{H}), 7.19$ $(\mathrm{m}, 2 \mathrm{H}), 3.50\left(\mathrm{AB}, J=16.2 \mathrm{~Hz}, 2 \mathrm{H}, \mathrm{SCH}_{2}\right), 2.97 \& 2.82\left(\mathrm{AB}, J=17.3 \mathrm{~Hz}, 2 \mathrm{H}, \mathrm{CH}_{2}\right), 1.68(\mathrm{~s}$, $\left.3 \mathrm{H}, \mathrm{CH}_{3}\right), 1.49\left(\mathrm{~s}, 3 \mathrm{H}, \mathrm{CH}_{3}\right) .{ }^{13} \mathrm{C} \mathrm{NMR}\left(100.6 \mathrm{MHz}, \mathrm{CDCl}_{3}\right) \delta 149.1,148.6,135.9,134.3,130.3$, 128.6, 127.3, 126.8, 124.4, 117.3, 85.8, 53.1, 33.4, 19.6, 19.4. HRMS Calcd for $\mathrm{C}_{18} \mathrm{H}_{20} \mathrm{NO}_{3} \mathrm{~S}_{2}$ $(\mathrm{M}+\mathrm{H})$ 362.0885. Found 362.0888. IR (neat) 1582, 1434, 1310, 1148, 1082, 1059, 877, 845, 797 , $746,715,688 \mathrm{~cm}^{-1}$. 
Crystal data of (7d). Bruker Kappa APEXII CCD diffractometer $\left(\mathrm{Mo}_{\mathrm{K} \alpha} \lambda=0.71073 \AA\right.$; graphite monochromator; $T=291(2) \mathrm{K})$. Formula $\mathrm{C}_{18} \mathrm{H}_{19} \mathrm{NO}_{3} \mathrm{~S}_{2}$, formula weight 361.46 , crystal system orthorhombic, space group Pca2 ${ }_{1}$, crystal dimensions $0.48 \times 0.38 \times 0.28 \mathrm{~mm}^{3}, a=13.311(4)$, $b=8.819(2), c=15.078(6) \AA, \alpha=\beta=\gamma=90.00^{\circ}, V=1769.9(10) \AA^{3}, Z=4, \rho_{\text {calcd }}=1.357 \mathrm{Mgm}^{-3}, \mu=0.32$ $\mathrm{mm}^{-1}, 2 \theta_{\max }=63.26^{\circ}, 47345$ measured reflections, 5897 independent reflections $\left(R_{\text {int }}=0.024\right), R 1$ $[I>2 \sigma(I)]=0.032, w R 2[I>2 \sigma(I)]=0.090, \mathrm{GOF}=1.03,219$ parameters, final difference map within 0.35 and $-0.20 \mathrm{e}^{-3}$. The structure was solved using direct methods and refined by full-matrix least-squares analysis on $F^{2}$. Numerical absorption corrections were applied (G. M. Sheldrick SHELXTL, Bruker ACS Inc.: Madison, WI, 2008). CCDC 784818 contains the supplementary crystallographic data for this paper. These data can be obtained free of charge from The Cambridge Crystallographic Data Center via www.ccdc.cam.ac.uk/data_request/cif. Selected bond lengths $(\AA)$ and angles (deg): S1-O2 1.4345(12), S1-O3 1.4418(13), S1-C1 1.8282(15), S2O1 1.4841(16), S2-C1 1.8677(13), C3-C4 1.334(2), C2-C3-C4 124.30(14), C3-C4-C5 122.75(14), C8-C1-S2 115.68(9), C2-C1-S2 102.97(9), S1-C1-S2 103.45(6).

2-Phenylsulfonyl-3,6-dihydro-4,5-dimethyl-1-oxo-2H-thiopyran-2-(2-quinoline)

(7e). Prepared by the general procedure from 2-phenylsulfonylmethylquinoline 5e and 2,3-dimethyl1,3-butadiene, using 2,6-lutidine as a base. Flash chromatography (pentane/EtOAc, 2:1 to 1:2) afforded $0.305 \mathrm{~g}$ (74\% yield) of a white powder, mp $181{ }^{\circ} \mathrm{C} .{ }^{1} \mathrm{H} \mathrm{NMR}\left(400.13 \mathrm{MHz}, \mathrm{CDCl}_{3}\right) \delta$ $8.21(\mathrm{~d}, J=11.4 \mathrm{~Hz}, 1 \mathrm{H}), 7.88(\mathrm{~d}, J=17.5 \mathrm{~Hz}, 1 \mathrm{H}), 7.15-7.35(\mathrm{~m}, 7 \mathrm{H}), 7.42(\mathrm{~m}, 2 \mathrm{H}), 3.66(\mathrm{AB}$, $J=17.4 \mathrm{~Hz}, 1 \mathrm{H}, \mathrm{CHH}), 3.27 \& 3.09\left(\mathrm{AB}, J=17.5 \mathrm{~Hz}, 2 \mathrm{H}, \mathrm{CH}_{2}\right), 2.90(\mathrm{AB}, J=17.4 \mathrm{~Hz}, 1 \mathrm{H}$, $\mathrm{CH} \underline{\mathrm{H}}), 1.60\left(\mathrm{~s}, 3 \mathrm{H}, \mathrm{CH}_{3}\right), 1.46\left(\mathrm{~s}, 3 \mathrm{H}, \mathrm{CH}_{3}\right) ;{ }^{13} \mathrm{C} \mathrm{NMR}\left(100.6 \mathrm{MHz}, \mathrm{CDCl}_{3}\right) \delta 152.3,146.8$, $136.8,135.4,134.3,130.8,130.0,129.2,128.4,127.8,127.6,127.5,124.9,120.6,116.5,85.5$, 51.9, 25.6, 20.8, 19.1. HRMS Calcd for $\mathrm{C}_{22} \mathrm{H}_{22} \mathrm{NO}_{3} \mathrm{~S}_{2}(\mathrm{M}+\mathrm{H})$ 412.1041. Found 412.1022. IR (neat) $1501,1310,1146,1082,1060,832,803,759,691 \mathrm{~cm}^{-1}$.

\section{Acknowledgements}

We gratefully acknowledge for financial support the Ministère de la Recherche et des Nouvelles Technologies (grant to H.D.), the 'CRUNCHOrga' (Centre de Recherche Universitaire Normand de Chimie Organique), the Région Basse-Normandie, the Centre National de la Recherche Scientifique (CNRS), and the European Union (FEDER funding). K. Jarsalé (University of Caen) is acknowledged for the mass analyses. Pr. A.-C. Gaumont, who is the leader of our research group in Caen, is acknowledged for helpful discussions. 


\section{References}

1. (a) Vyas, D. M.; Hay, G. W. J. Chem. Soc., Perkin Trans. 1 1975,180. (b) Adam, D.; Freer, A. A.; Isaacs, N. W.; Kirby, G. W.; Littlejohn A.; Rahman, M. S. J. Chem. Soc., Perkin Trans. I, 1992, 1261. (c) Herczegh, P.; Zsely, M.; Bognar, R.; Szilagyi, L. Tetrahedron Lett. 1986, 27, 1509. (e) Vedejs, E.; Galante, R. J.; Goekjian, P. G. J. Am. Chem. Soc. 1998, 120, 3613. (f) Prabharakan, J.; Lhermitte, H.; Das, J.; Sasi-Kumar, T. K.; Grierson, D. S. Synlett 2000, 658. (g) Pinto, I. L.; Buckle, D. R.; Rami, H.; K Smith, D. G. Tetrahedron Lett. 1992, 33, 7597. (h) Heras, M.; Gulea, M.; Masson, S. Chem. Commun. 2001, 611. (i) Heras, M.; Gulea, M.; Masson, S.; Philouze, C. Eur. J. Org. Chem. 2004, 160. (j) Bastin, R.; Albadri, H.; Gaumont, A-C.; Gulea, M. Org. Lett. 2006, 8, 1033. (k) Watanabe, Y.; Sakokibara T. Tetrahedron 2009, 65, 599.

2. See as reviews dealing with thiosugars: (a) Robina, I.; Vogel, P.; Witczak, Z. J. Curr. Org. Chem. 2001, 5, 1177. (b) Witczak, Z. J. Curr. Med. Chem. 1999, 6, 165.

3. (a) Denancé, M.; Legay, R.; Gaumont, A.-C.; Gulea, M. Tetrahedron Lett. 2008, 49, 4329.

(b) Yoshimura, Y.; Yamazaki, Y.; Saito, Y.; Takahata, H. Tetrahedron 2009, 65, 9091.

4. Dentel, H. and Gulea, M., unpublished results.

5. For a review on sulfines chemistry, see: Zwanenburg, B.; Damen, T. J. G.; Philipse, H. J. F.; De Laet, R. C.; Lucassen, A. C. B. Phosphorus, Sulfur and Silicon 1999, 153-154, 119, and references cited therein.

6. Lempereur, C.; Ple, N.; Turck, A.; Queguiner, G.; Corbin, F.; Alayrac, C.; Metzner, P. Heterocycles 1998, 48, 2019.

7. Lucassen, A. C. B.; Zwanenburg, B. Eur. J. Org. Chem. 2004, 74.

8. See also for another method, via a retro Diels-Alder reaction: Capozzi, G.; Fratini, P.; Menichetti, S.; Nativi, C. Tetrahedron 1996, 52, 12133.

9. Gajda, T. Synthesis 1988, 327.

10. Abrunhosa, I.; Gulea, M.; Masson, S. Synthesis 2004, 6, 928.

11. This geometry was found by $\mathrm{X}$-ray diffraction analysis in the case of the sulfonyl-sulfenylsulfine $\mathrm{C}_{6} \mathrm{Cl}_{5} \mathrm{~S}(\mathrm{C}=\mathrm{S}=\mathrm{O}) \mathrm{SO}_{2}$ Tol): El-Sayed, I. Sulfur Lett. 1999, 23, 1.

12. (a) Liu, X.-L.; Sheng, S.-R.; Zhou, W.; Wang, Q. Y.; Zhang, X.-L.; Gong, B. Synthetic Commun. 2007, 37, 119. (b) Jang, W. B.; Jeon, H. J.; Oh, D. Y. Synthetic Commun. 1998, $28,1253$. 